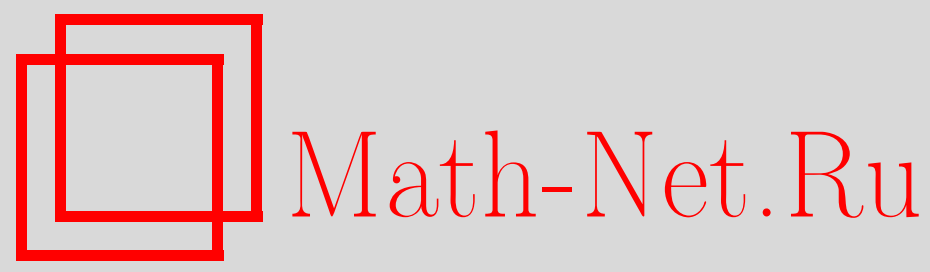

В. М. Шелкович, Теория обобщенных функций Коломбо, использующая гармонические регуляризации, Матем. заметки, 1998, том 63, выпуск 2, 313-316

DOI: https://doi.org/10.4213/mzm1284

Использование Общероссийского математического портала Math-Net.Ru подразумевает, что вы прочитали и согласны с пользовательским соглашением http://www.mathnet.ru/rus/agreement

Параметры загрузки:

IP: 3.80 .253 .173

26 апреля 2023 г., $14: 15: 41$

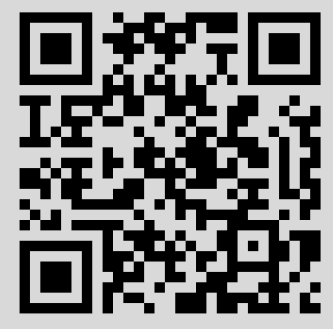




\section{ТЕОРИЯ ОБОБЩЕННЫХ ФУНКЦИЙ КОЛОМБО, ИСПОЛЬЗУЮЩАЯ ГАРМОНИЧЕСКИЕ РЕГУЛЯРИЗАЦИИ}

\section{В.М. Шелкович}

Введение. Более десяти лет назад Ж. Коломбо [1] построил ассоциативную алгебру новых обобщенных функций, в которую вкладывалось пространство распределений Шварца. Произведения распределений в общем случае являлись новьми обобщенными функциями. Связь новых обобщенных функций с распределениями осуществлялась через понятие ассоциированного распределения или асимптотики. Используемая при вложении пространства распределений регуляризация (см. [1], [2]) делает весьма затруднительньм вычисление асимптотик и использование теории в приложениях.

Мы строим версию теории Коломбо, в которой для вложения пространства распределений используется гармоническая (точнее полигармоническая) регуляризация. Такой подход позволяет легко находить асимптотические разложения произведений распределений и применять теорию в аналитических приложениях. В [3], [4] была продемонстрирована эффективность гармонических регуляризаций для приложений. Данная задача упоминается в книге [2, задача 27.3] в списке нерешенных проблем. В нашу алгебру инъективно вкладьвается подалгебра распределений из [3], [4], элементы которой являются вектор-распределениями.

1. Алгебра Коломбо. Пусть $\mathscr{E}_{M}$ - дифференциальная алгебра бесконечно дифференцируемых функций-последовательностей $f_{m}(x, y)$, где $m \in \mathbb{N}, \mathbb{N}$-множество натуральных чисел, $y>0$ и $x \in \mathbb{R}^{n}$, умеренного роста: для любого $K \subset \mathbb{R}^{n}$ и любого $D^{k}=\frac{\partial^{|k|}}{\partial x_{1}{ }^{k} \cdots \partial x_{n}{ }^{k_{n}}}$ найдутся $p, m \in \mathbb{N}$
и константа $M>0$ такие, что при некотором $\varepsilon_{0}>0$

$$
\left|D^{k} f_{m}(x, y)\right|<M y^{-p}, \quad x \in K, \quad y \in\left(0, \varepsilon_{0}\right) .
$$

Пусть $\mathscr{N}$ - множество элементов $f_{m}(x, y) \in \mathscr{E}_{M}$ таких, что для любых $K \subset \mathbb{R}^{n}, D^{k}$ и $p \in \mathbb{N}$ существуют число $m=m(K, k, p) \in \mathbb{N}$ и константа $M>0$, что при некотором $\varepsilon_{0}>0$

$$
\left|D^{k} f_{m}(x, y)\right|<M y^{p}, \quad x \in K, \quad y \in\left(0, \varepsilon_{0}\right) .
$$

Очевидно, что $\mathscr{N}$ - идеал в алгебре $\mathscr{E}_{M}$. Элементы факторпространства (факторалгебры) $\mathscr{G}=$ $\mathscr{E}_{M} / \mathscr{N}$ назовем обобш,енными функииями Коломбо.

Мы привели один из стандартных вариантов конструкции Коломбо [1, гл. 1], [2, III], [5], [6], где, как правило, элементы $\mathscr{E}_{M}$ зависят как от параметра, так и от функции $\psi \in D\left(\mathbb{R}^{n}\right), D=D\left(\mathbb{R}^{n}\right)$ пространство основных функций с компактным носителем.

Вложение $D^{\prime}$ и $C^{\infty}$ в $\mathscr{G}$ осуществляется при помощи регуляризации Коломбо

$$
f_{\psi, y}(x)=\left\langle f(\xi), \frac{1}{y^{n}} \psi\left(\frac{\xi-x}{y}\right)\right\rangle \in \mathscr{E}_{M}, \quad x \in \mathbb{R}^{n}
$$

где $\psi(x)=\prod_{i=1}^{n} \chi\left(x_{i}\right) \in D\left(\mathbb{R}^{n}\right)$ и $\chi\left(x_{i}\right) \in D(\mathbb{R}), i=1, \ldots, n$ (подробнее см. [1], [2], [5], [6]).

Регуляризации $\delta$-функции и функции Хевисайда $\theta(x)$ могут иметь вид

$$
\delta_{\psi, y}(x)=y^{-n} \psi\left(\frac{-x}{y}\right), \quad \theta_{\psi, y}(x)=\int_{\mathbb{R}^{n}} y^{-n} \psi\left(\frac{\xi-x}{y}\right) d \xi
$$

Ясно, что поиск соотношений, связьвающих такие представления, весьма затруднителен.

Работа выполнена при финансовой поддержке Российского фонда фундаментальных исследований, гранты № 95-01-01084 и № 96-01-01199а. 
2. Полигармонические представления распределений. Пусть $\mathbb{R}_{+}^{n+1}=\{(x, y) \in$ $\left.\mathbb{R}^{n+1}: x \in \mathbb{R}^{n}, y>0\right\}$. Обозначим через $G=G\left(\mathbb{R}_{+}^{n+1}\right)$ пространство функций $f(x, y)$ :

1) гармонических в $\mathbb{R}_{+}^{n+1}$, т.е. $\Delta f(x, y)=0, \Delta=\sum_{k=1}^{n+1} \frac{\partial^{2}}{\partial x_{k}^{2}}-$ оператор Лапласа, $x_{n+1}=y$;

2) имеющих конечный порядок роста при подходе к $\mathbb{R}^{n}$, т.е. если для любого компакта $K \subset \mathbb{R}^{n}$ найдутся константы $C>0, \alpha>0$, для которых

$$
|f(x, y)| \leqslant C y^{-\alpha}, \quad y \in(0,1], \quad x \in K .
$$

Теорема 1 [7, следствие 11.9]. Всякая функиия $f(x, y) \in G\left(\mathbb{R}_{+}^{n+1}\right)$ определяет на $\mathbb{R}^{n}$ некоторое распределение $f(x) \in D^{\prime}\left(\mathbb{R}^{n}\right)$ :

$$
\lim _{y \rightarrow+0}\langle f(x, y), \varphi(x)\rangle=\langle f(x), \varphi(x)\rangle \quad \forall \varphi(x) \in D\left(\mathbb{R}^{n}\right) .
$$

Для всякого распределения $f(x) \in D^{\prime}\left(\mathbb{R}^{n}\right)$ найдется функиия $f(x, y) \in G\left(\mathbb{R}_{+}^{n+1}\right)$, для которой выполняется равенство (3). Причем $f(x, y) \in C^{\infty}\left(\mathbb{R}_{+}^{n+1} \cup\left(\mathbb{R}^{n} \backslash \operatorname{supp} f(x)\right)\right)$ ина $\mathbb{R}^{n} \backslash \operatorname{supp} f(x)$ функиия $f(x, y)=0$.

Пусть $G_{m}=G_{m}\left(\mathbb{R}_{+}^{n+1}\right), m=1,2, \ldots,-$ пространство функций $f(x, y)$ :

1) полигармонических (m-гармонических) в $\mathbb{R}_{+}^{n+1}$, т.е. $\Delta^{m} f(x, y)=0$;

2) имеющих конечный порядок роста при подходе к $\mathbb{R}^{n}$.

Из (1) и (2) следует, что $G_{m}\left(\mathbb{R}_{+}^{n+1}\right) \subset \mathscr{E}_{M}$ для любого $m$.

ТЕорема 2. Для того чтобъ функиия $f(x, y) \in G_{m}\left(\mathbb{R}_{+}^{n+1}\right)$, необходимо и достаточно, чmoбbl

$$
f(x, y)=\sum_{k=0}^{m-1} y^{k} f_{k}(x, y)
$$

әде $f_{k}(x, y) \in G\left(\mathbb{R}_{+}^{n+1}\right)$ - гармонические функчии, имеющие конечный порядок роста.

ДокАЗАТЕЛЬСтво. Необходимость следует из [7, лемма 12.8]. Докажем достаточность, применяя к (4) формулу из [7, 12.7$]$ :

$$
\Delta f(x, y)=\sum_{j=0}^{m-3} y^{j}(j+1)\left((j+2) f_{j+2}+2 \frac{\partial f_{j+1}}{\partial y}\right)+2 y^{m-2}(m-1) \frac{\partial f_{m-1}}{\partial y} .
$$

Используя для последнего выражения $\Delta^{m-1}$, получим утверждение.

Из теорем 1,2 и следствия 12.9 из [7] вытекает, что любая функция $f(x, y) \in G m\left(\mathbb{R}_{+}^{n+1}\right)$ определяет на $\mathbb{R}^{n}$ некоторое распределение $f(x) \in D^{\prime}\left(\mathbb{R}^{n}\right)$, т.е. имеет место (3).

ОПредЕЛЕНИЕ. Пусть $f(x) \in D^{\prime}\left(\mathbb{R}^{n}\right)$ - распределение, $f(x, y)$ - его гармоническая аппроксимация. Функцию

$$
f_{(m)}(x, y)=\sum_{s=0}^{m-1} \frac{(-1)^{s}}{s !} y^{s} D_{y}^{s} f(x, y) \in G_{m}\left(\mathbb{R}_{+}^{n+1}\right)
$$

назовем канонической $m$-гармонической аппроксимачией распределения $f(x), m=1,2, \ldots$

ЛЕмма 1. Пусть $f(x) \in D^{\prime}\left(\mathbb{R}^{n}\right)$ - распределение, $f_{(m)}(x, y) \in G_{m}\left(\mathbb{R}_{+}^{n+1}\right)$ - его каноническая $m$-гармоническая аппроксимачия. Тогда для любого $m \in \mathbb{N}$ имеем в слабом смислле $f_{(m)}(x, y)=f(x)+o\left(y^{m-1}\right), y \rightarrow+0$. 
ДоКАЗАТЕЛЬСТвО. Представим все члены (5) с помощью формулы Тейлора по $y \in\left(0, \varepsilon_{0}\right)$ при $s=0,1, \ldots, m-1$ :

$$
y^{s} D_{y}^{s} f(x, y)=\sum_{k=0}^{m-1-s} \frac{1}{k !} y^{k+s} D_{y}^{k+s} f(x, 0)+\frac{1}{m !} y^{m} D_{y}^{m} f\left(x, \theta_{s} y\right), \quad 0<\theta_{s}<1 .
$$

Здесь $D_{y}^{m} f\left(x, \theta_{s} y\right) \in G\left(\mathbb{R}_{+}^{n+1}\right), D_{y}^{j} f(x, 0) \in D^{\prime}\left(\mathbb{R}^{n}\right)$ - распределения, определяемые как

$$
\left\langle D_{y}^{j} f(x, 0), \varphi(x)\right\rangle=\lim _{y \rightarrow+0}\left\langle D_{y}^{j} f(x, y), \varphi(x)\right\rangle \quad \forall \varphi(x) \in D,
$$

и $f(x, 0)=f(x)$. Используя эти представления, из (5) получим

$$
f_{(m)}(x, y)=f(x)+\sum_{k=1}^{m-1} \sum_{s=0}^{k} \frac{(-1)^{s}}{s !(k-s) !} y^{k} D_{y}^{k} f(x, 0)+y^{m} \sum_{s=0}^{m-1} \frac{(-1)^{s}}{s !(m-s) !} D_{y}^{m} f\left(x, \theta_{s} y\right) .
$$

Так как

$$
\sum_{s=0}^{k} \frac{(-1)^{s}}{s !(k-s) !}=0
$$

получим, что

$$
f_{(m)}(x, y)=f(x)+y \sum_{s=0}^{m-1} \frac{(-1)^{s}}{s !(m-s) !} D_{y}^{m} f\left(x, \theta_{s} y\right) .
$$

Учитывая, что при достаточно малых $y>0$ для $s=1, \ldots, m-1$

$$
\left|\left\langle D_{y}^{m} f\left(x, \theta_{s} y\right), \varphi(x)\right\rangle\right| \leqslant C \quad \forall \varphi(x) \in D\left(\mathbb{R}^{n}\right),
$$

где $C>0$ - некоторая константа, из (6) получим утверждение леммы.

Если $f(x) \in \mathscr{E}^{\prime}\left(\mathbb{R}^{n}\right)$ (или $\left.\mathscr{O}_{-n}^{\prime}\left(\mathbb{R}^{n}\right)\right)$, то его каноническую $m$-гармоническую аппроксимацию можно получить с помощью $m$-интегрального представления Пуассона:

$$
f_{(m)}(x, y)=\sum_{s=0}^{m-1} \frac{(-1)^{s}}{s !} y^{s} D_{y}^{s} f(x, y)=\left\langle f(t), P_{m}(x-t, y)\right\rangle, \quad y>0, \quad m \geqslant 1 .
$$

Ядро интегрального представления (7) имеет вид

$$
P_{m}(x, y)=\frac{\Gamma((n+1) / 2)}{\pi^{(n+1) / 2}} \sum_{j=0}^{m-1} \frac{(-1)^{j}}{j !} y^{j} D_{y}^{j}\left(\frac{y}{\left(|x|^{2}+y^{2}\right)^{(n+1) / 2}}\right),
$$

где $\Gamma(x)$ - гамма-функция. Интегральное представление (7) получается из представления Пуассона $[7, \S 11, \S 12]$ (см. $[8,13.5])$ с использованием леммы 1.

Применяя к (7) формулу Тейлора, аналогично лемме 1 получим, что если $f(x) \in C^{\infty}\left(\mathbb{R}^{n}\right)$, то для любого $m \in \mathbb{N}$ и любого компакта $K \subset \mathbb{R}^{n} f_{(m)}(x, y)=f(x)+o\left(y^{m-1}\right), y \rightarrow+0$, где $f_{(m)}(x, y)$-каноническая $m$-гармоническая аппроксимация $(7)$.

3. Вложения $C^{\infty} \subset \mathscr{G}$ и $D^{\prime} \subset \mathscr{G}$. Вложения пространств $C^{\infty}$ и $D^{\prime}$ в алгебру Коломбо проведем аналогично $[1],[2, \S 9],\left[3\right.$, п. 6, п. 7] с помощью отображения $f(x) \mapsto f_{(m)}(x, y) \in \mathscr{E}_{M}$.

Лемма 2. Вложение $C^{\infty} \subset \mathscr{G}$ - аләебраический изоморфизм. 
ДоКАЗАТЕльство. В алгебре Коломбо $\mathscr{G}$, как подмножество, имеется пространство $C^{\infty}$, элементы которого суть классы функций вида $f_{(m)}(x, y)=f(x)$ для любых $m \in \mathbb{N}, y>0$, где $f(x) \in C^{\infty}$. Тогда согласно лемме 1 для любого $K \subset \mathbb{R}^{n}$ и любого $m \in \mathbb{N}$

$$
g_{(m)}(x, y)=f(x)-f_{(m)}(x, y)=o\left(y^{m-1}\right), \quad y \rightarrow+0, \quad x \in K
$$

Таким образом, $f(x)-f_{(m)}(x, y) \in \mathscr{N}$.

Лемма 3. Пусть $f(x) \in \mathscr{E}^{\prime}\left(\mathbb{R}^{n}\right)\left(\right.$ или $\left.\mathscr{O}_{-n}^{\prime}\left(\mathbb{R}^{n}\right)\right)-$ распределение, $f_{(m)}(x, y)-$ его каноническая т-гармоническая аппроксимачия (7). Тогда $f(x)=0$ в том и только том cлучае, когда $f_{(m)}(x, y) \in \mathcal{N}$.

Доказательство проводится как в лемме 4.10 из [5]. Несложно обобщить лемму 3 на случай

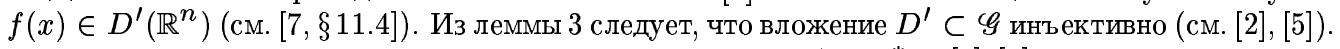

Можно показать, что ассоциативно-коммутативная алгебра $E^{*}$ из [3], [4], порожденная распределениями $\delta^{(m-1)}(x), P\left(x^{-m}\right)$ и мультипликаторами $x^{m-1}, m=1,2, \ldots$, инъективно вкладывается в $\mathscr{G}$ при $n=1$.

\section{СПИСОК ЦИТИРОВАННОЙ ЛИТЕРАТУРЫ}

1. Colombeau J. F. Elementary Introduction to New Generalized Functions. Amsterdam: North-Holland, 1985. 2. Oberguggenberger M. Multiplication of Distributions and Applications to Partial Differential Equations. New York, 1992. 3. Шелкович В. М. // Докл. АН СССР. 1990. Т. 314. № 1. С. 159-164. 4. Шелкович В. М. // Матем. заметки. 1995. Т. 57. № 5. С. 765-783. 5. Todorov T. D. Sequentional Approach to Colombeau's Theory of Generalized Functions. Preprint IC/87/26. Trieste: ICТР, 1987. 6. Егоров Ю. В. // УМН. 1990. Т. 45. № 5. С. 3-40. 7. Кытманов А. М. Интеграл Мартинелли-Бохнера и его применения. Новосибирск: Наука, 1992. 8. Бремерман Г. Распределения, комплексные переменные и преобразования Фурье. М.: Мир, 1968. 